\title{
Application of Systems Thinking to Energy Demand Reduction
}

\author{
Rachel Freeman, Theo Tryfonas \\ Systems Centre, University of Bristol \\ Merchant Ventures Building, Woodland Road, \\ Bristol BS8 1UB, UK \\ email: cerajf@bristol.ac.uk, theo.tryfonas@bristol.ac.uk
}

\begin{abstract}
The need to reduce energy demand has been firmly established, but progress so far has been limited. This paper postulates that a Systems Thinking approach could address some of the weaknesses of current policies, which include a lack of deep understanding into what really drives energy demand and how to change it at the user level. Several systems analysis tools are reviewed: the energy system can be conceptualized as a sociotechnical regime within a Multi Level Perspective framework, with regime changes a result of landscape pressures; alternatively, subsystems within the demand side system can be classified by problem-context type. Regarding applications of systems methodologies, a case study into the use of systems methods at Sustain Ltd by their lowcarbon buildings practice is given. A non-capital approach that combines technology optimization, behavior change, and information feedback is expected to produce quick and low-cost energy savings within a school.
\end{abstract}

Keywords: energy demand reduction, carbon emissions reduction, systems thinking, sociotechnical transitions

\section{Introduction}

The UK's Climate Change Act of 2008 set into law firm carbon reduction targets for 2020 and 2050, and the Department of Energy and Climate Change's Low Carbon Transition Plan states that 'reducing our demand for energy from the energy system is fundamental to the Government's strategy'[2]. The plan includes requirements for reductions in energy demand of between $26 \%$ and $43 \%$ by 2050 compared to 2005 levels, which could be achieved 'primarily through energy efficiency, but there will also be some energy demand reduction from substitution to less energy intensive activities and less waste of energy'. [2]

The grid-based energy system that our modern society depends upon is a complex web of supply and demand that stretches over the whole country. This paper focuses exclusively on the demand side of that system, which consists of energy used in buildings. We refer to it as the 'energy demand system' - the part of the energy system that lives 'behind the meter'. This paper does not consider transport-related energy use or the supply side, which includes central power generation and transmission and distribution of power.

The energy demand system is highly diverse across sectors, multi-layered, highly influenced by the psychology and sociology of people, and complex in the diversity of applications of energy-using technologies - in other words, reducing demand is a 'wicked' problem [3]. This is illustrated by the fact that although energy efficiency is being implemented on a wide scale throughout the UK, in 2009 energy demand in the transport and residential sectors was $21 \%$ and $13 \%$ higher, respectively, than 1990 levels, and domestic per capita consumption was only $1 \%$ lower than in 1990. [4]

Up to now, demand reduction interventions have mostly sought to address single issues such as end-use equipment efficiency and operation, energy behaviors, or distributed generation, but there have been calls for a more integrated approach. Wilson and Dowlatabadi envisage a goal of entrenching the 'social and behavioral determinants of energy use as a wholly integrated part of energy efficiency research' [5] which indicates a systems approach of some kind. This paper argues that many of the methodologies and concepts in the field of Systems Thinking (ST) are appropriate tools when working on the demand side. There are two separate but related research questions which ST could be used to help answer:

1. What is the nature of energy demand in society, can the energy demand system be understood as a whole, and can we identify how transitions happen within it? 
2. Can we develop better interventions to reduce energy demand at the user level, which see each household or organization as a system that includes technology, buildings and people and work with the interactions between these components?

The paper concludes with a case study into the use of ST by consultants at Sustain Limited to assist a variety of clients in reducing their carbon emissions.

\section{Modeling the Energy Demand System as a Whole}

The first research question identified above - the nature of energy demand - could be explored by conceptualizing the energy demand system as a system model. However, this is an open system that interacts with and is affected by all components of society and technology, so there are few obvious and clearly defined boundaries. Therefore, the first step is to identify a System of Interest (SoI).

\subsection{The System of Interest}

The SoI selected for this research is defined as the domestic and commercial built environment. Figure I shows this SoI within its larger operating environment.

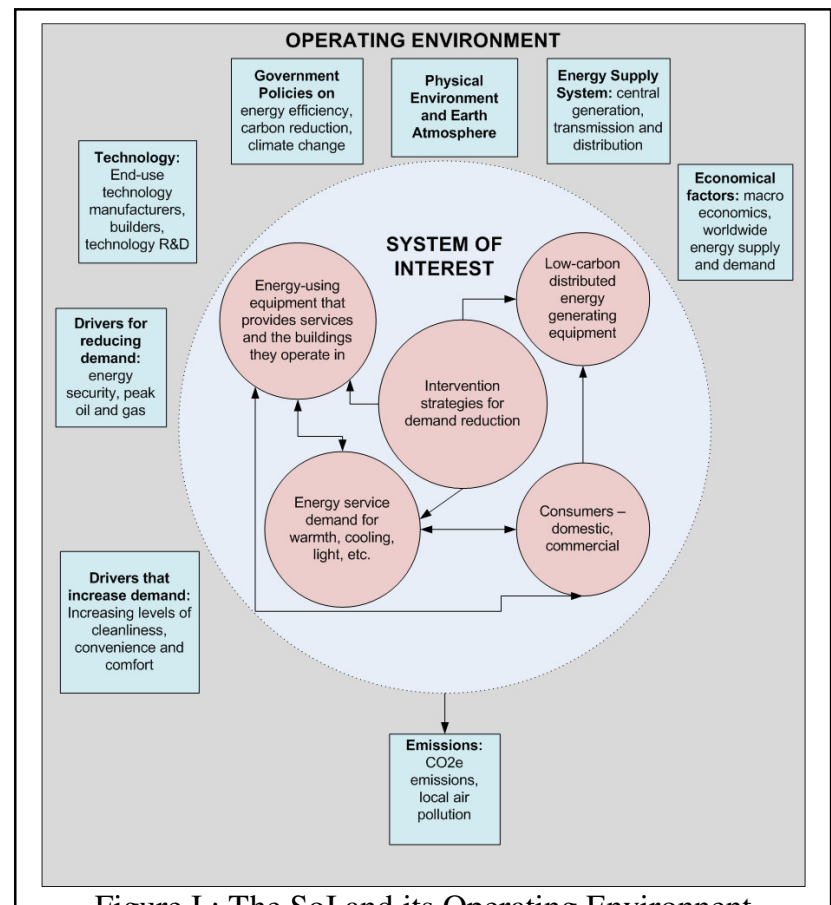

Figure I : The SoI and its Operating Environnent

- Within the system boundary there are five main categories of components: people (consumers), energy service demand (need for warmth, light, motor power, etc.), energy-using equipment that provides services (boilers, light bulbs etc.), low-carbon energy generating equipment (solar PV, CHP, etc.), and intervention strategies (energy behavior change, energy efficiency upgrades, etc).

- The SoI sits within the Operating Environment of wider society, made up of many components which affect how it operates, as well as providing inputs that allow the system to function. The outputs from the SoI are the emissions associated with energy use. This is the metric that interventions are seeking to reduce.

It will be helpful to analyze the SoI within a conceptual framework, and one such example is presented below.

\subsection{The Multi-Level Perspective}

The Multi-Level Perspective (MLP), as described by Geels and Schot [1] and shown in Figure II, is a threelayered framework that is large enough to represent both the whole SoI and its exogenous environment.

Within this model, the demand-side system can be

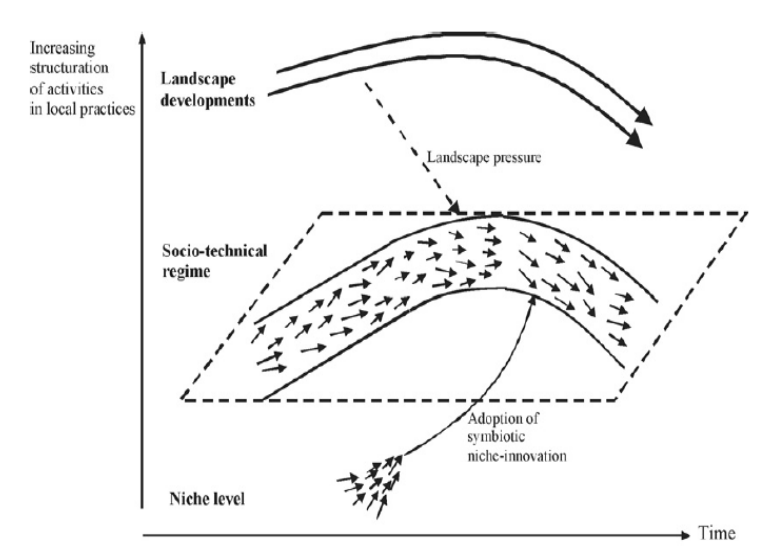

Figure II: The MLP and Transformation Pathways [1]

seen as a 'sociotechnical regime' that lives within the environment of the exogenous 'sociotechnical landscape'. The sociotechnical landscape is equivalent to the operating environment as shown in Fig I. The regime is equivalent to the SoI we have already defined. It represents the fixed structure of the system, with technology development trajectories set in a pattern and only incremental changes to technology pursued, as opposed to fundamental ones. Thus, equipment manufacturers may build more efficient versions of their energy-using products but they will not think of new ways to achieve the desired end-user service. On the third level are niche innovations. Niche innovators will find new ways to meet a service need, rather than making incremental changes to existing ideas. From time to time these niche innovations can penetrate the established regime; however certain conditions are required, such as the need for the established regime to change due to downward pressures coming from disruptions in the 
landscape, and the suitability of new technologies to meet that need.

The MLP goes much further than a simple systems diagram in that it can help to reveal how systems transform over time. Its applicability to the energy demand system is confirmed by Geels when he states that "climate change may in future decades become such a disruptive landscape change, triggering such a sequence of transition paths in transport and energy regimes'. This high-level approach could help to answer questions about the underlying and sometimes hidden drivers of energy demand, which have so far been difficult to identify. Wilhite et al. argue that demand is manufactured and is primarily a social construct. They call for new research that approaches the dynamics of energy demand as an understanding of 'sociotechnical change and the co-evolution of infrastructures, devices, routines and habits.' [6] This emphasis on sociotechnical change chimes well with the MLP and its ability to model how transitions happen within sociotechnical regimes. Specifically, the MLP could be used to gain insight into the effects of overlying and long-term social, political, technical, or macro-economic influences on the demand in the energy system as a whole.

\section{Identifying and Working with Subsystems}

In order to answer the second research question presented in the introduction, we need to bring the analysis to a much more detailed level and identify the subsystems that can be worked with. At this level the system boundary would be around a single household or organization, and the components of the system are of two types: hard or soft. Hard subsystems are the physical building(s) and energy using equipment in them; soft subsystems are the (one or more) collection of people that buy and operate that equipment. Presented here are ways to identify subsystems and some of the ST methods that could be used to work with them.

\subsection{System of Systems Methodologies}

There are many different ST methodologies, developed in and suited to particular types of systems or problem spaces. Jackson and Keys developed a way of categorizing both problem contexts and the methodologies suitable for them in their 'system of systems methodologies' [7]. Within this classification scheme systems are either mechanical (relatively easy to understand), or systemic (manifesting difficult problems). Participants are unitary if they all agree on a common set of goals, and pluralist if they have differing objectives. All problem contexts can be assigned to one of four categories: mechanical-unitary, systemic-unitary, mechanical-pluralist, or systemic-pluralist.
Care must be taken in using this categorization system due to the danger of it leading to the application of a method from one paradigm to a problem space that exists in another [8]. However, the approach is a useful tool in identifying and classifying subsystems within the larger SoI. Two examples follow.

- A house's heating system is mechanical-unitary - the system components are well known and the users have a common objective (maintain comfortable indoor temperature throughout the house).

- A school and its stakeholders (including the buildings, staff and pupils, facilities management, and outside interests such as Local Authority) is systemicpluralist - participants may have differing priorities as far as use of equipment and the buildings are concerned (energy costs, indoor comfort, convenience, educational needs, etc.). The system is complex and has emergent properties - like when post-occupancy energy demand is higher than was planned for during the design stage by architects and builders.

\subsection{Improving Energy Subsystems Using Systems Engineering}

Hard subsystems - for example, heating and lighting systems in buildings; motors and appliances; the building envelope (windows, walls, etc.) - could be optimized with the use of a systems engineering (SE) approach. The benefits of SE are that it is inter-disciplinary, it enables complex systems to be modeled and organized, and it considers component interaction - like that between the interior layout of a building and its heating system performance. SE can be used in specific projects to identify synergies between different energy saving technologies, analyze technology-people interactions, do economic and energy tradeoffs, define system requirements, identify feedback loops, and determine when to use automated controls versus manual controls. For example, designers can either ask staff to turn lights off when not needed or install daylighting controls that ensure lighting only comes on when there is insufficient daylight.

\subsection{Soft Systems - Energy Behavior Change Using Soft Systems Methodologies}

The impact of how technology is used on overall energy consumption and building comfort can be quite large. For example, a report on developing low carbon schools states that "poor behavioral patterns and misuse may lead to the energy consumed in a school building being up to $45 \%$ higher than predicted.' [9] To understand how people interact with technology and make decisions about its use, different models of energy decision-making 
have been developed in the fields of psychology, conventional and behavioral economics, technology diffusion, and the social sciences. However, because of the heterogeneity of energy decision making throughout the whole population, these models may apply only in specific behavioral niches - determined by where they live on the individual-to-social, instinctive-to-deliberate, psychological-to-contextual, and short-to-long term continua. [5] Based on these behavior models, various types of energy behavior change (BC) campaigns have been run with genuine but modest effects proven; evidence points to increased effectiveness when BC campaigns are targeted towards specific sub-groups and the need for consistent messaging.

Within an organization, there are several softsystems-oriented methodologies that could be applied to achieve energy BC. They can be differentiated between those based on ST (that rely on diagramming and analysis) versus those that work in a systemic way (that rely on action research and allow for emergence). Three examples are given here:

- Checkland's Soft Systems Methodology [10] is a ST method that establishes a learning system for investigating messy problems and enables practitioners to 'bring to the surface different perceptions of the problem and then structure these in a way that all involved find fruitful'. [11] It is most useful for collaborative behavior change within organizations.

- $\quad$ Kurtz and Snowden's Cynefin Framework [12]is a ST model applicable to complex adaptive systems and their inherent uncertainty. Cognitive Edge ${ }^{1}$ methods, based on this framework, are derived from complexity and narrative principles and include techniques such as Metaphor Simulations, Social Network Stimulation, and Archetypes. These methods are useful when working to evolve the functions of an organization.

- Action research is a systemic approach that allows for a looser exploration of organizations and how their operations transform. The LowCarbonWorks project [13] used action research to investigate four case studies of carbon reduction within organizations. Case study results were successfully mapped to the MLP, and tools for using action research to collaboratively achieve carbon reductions in the commercial sector were developed.

Although we have found no direct evidence that these three techniques are suitable tools when specifically

\footnotetext{
${ }^{1}$ www.cognitive-edge.com
}

targeting energy use in an organization, as opposed to more general optimization of operations (for which there is ample evidence), our hypothesis is that all three are flexible enough to be adapted to an energy-focused intervention strategy.

\section{Case Study: A ST Approach to Reducing Energy Demand in Schools}

Sustain Ltd. are a carbon reduction company that work with a wide range of clients, including housing associations, construction companies, manufacturers, and utilities. Their work includes the analysis of carbon emissions associated with buildings and manufactured products. Because Sustain's clients are very diverse there is a wide range of possible applicability for systems-oriented approaches but also a need to purposefully match the method with both the client and their specific problem.

Up to now Sustain's building services have focused on performing energy assessments for new buildings and energy audits for existing buildings. The client receives recommendations on a range of energy efficiency, low carbon generation and renewable technologies. Two recent trends are indicating the need for a more integrated approach. Firstly, many of the easy and cost-effective technology upgrades have already been done in nonresidential buildings, leading to an interest in softer interventions such as behavior change and stakeholder engagement. Secondly, buildings frequently do not perform at their design efficiency due to the way they are operated. This is an emergent property of the interaction between the soft and hard components of the system (i.e. people and technology) and it cannot be solved through technology alone.

There exist several integrated approaches to improving building performance, like the Soft Landings Protocol for new buildings [13] which deals with the uncertainty in post-occupancy building performance. Sustain have been developing their own systems-oriented, integrated methodology specifically designed for existing, occupied, commercial buildings, where a comprehensive technology retrofit is not an option - i.e. this is a low-cost and low-capital approach. The approach aims to achieve quick and low-cost energy savings without major disruption to daily operations, and possibly provide additional non-energy benefits such as improved overall sustainability performance for the organization. It combines three main types of interventions:

1. Energy information feedback, provided by a system of sensors throughout the building with real-time data displays, is a crucial tool that enables hidden energy wastage to be revealed and promotes a sense of agency 
in participants when they see the results of their actions.

2. Behavior change is essential for ensuring energy is a consideration during normal organizational operations. Methods include: end-user training on equipment use, developing a board-level energy policy and long-term energy plan, streamlining operations to promote efficient building use (e.g. equipment switch off policies), and energy awareness workshops for staff.

3. Technology controls: reduce energy wastage due to end-use equipment being badly maintained or controlled. Technologies such as automated controls can replace the need for manual controls and Building Management Systems can optimize building subsystems to work in synch with the organization's scheduling needs (e.g. heating is only on when the building is occupied). Policies can be put in place to ensure equipment is always switched off out of hours, either by facilities managers or by end users.

It is expected that applying these three interventions at the same time will make use of the positive synergies between them. In other words, this will be a system of interventions. Figure III portrays this approach as applied to a school. The diagram shows that the school is in fact part of a complex system, with influences on its operations coming from government educational policies, the $\mathrm{PFI}^{2}$, and the local authority. The smaller circles at the centre are the interventions to be applied to the system, and the red arrows show the expected synergies between the interventions. The key benefits of this approach are expected to be as follows:

- $\quad$ Providing energy feedback to both facilities managers and end users will enable them to track the effects of their efforts to save energy and also to identify where energy is being used unnecessarily. This should help ongoing behavior change efforts, especially when realtime dashboards are provided that give users an instant result for their efforts.

- $\quad$ Seeing the school as part of a larger system brings clarity to what is sometimes a confusing and complicated structure, as each stakeholder within the structure seeks to maintain his or her performance subject to the policies currently in place. For example, if annual energy savings goals are set against a baseline of the previous year's use, then it is in the facilities manager's interest to make energy savings more slowly rather than quicker.

\footnotetext{
${ }^{2}$ Performance Finance Initiative (PFI) companies build and maintain schools and then lease them to local authorities.
}

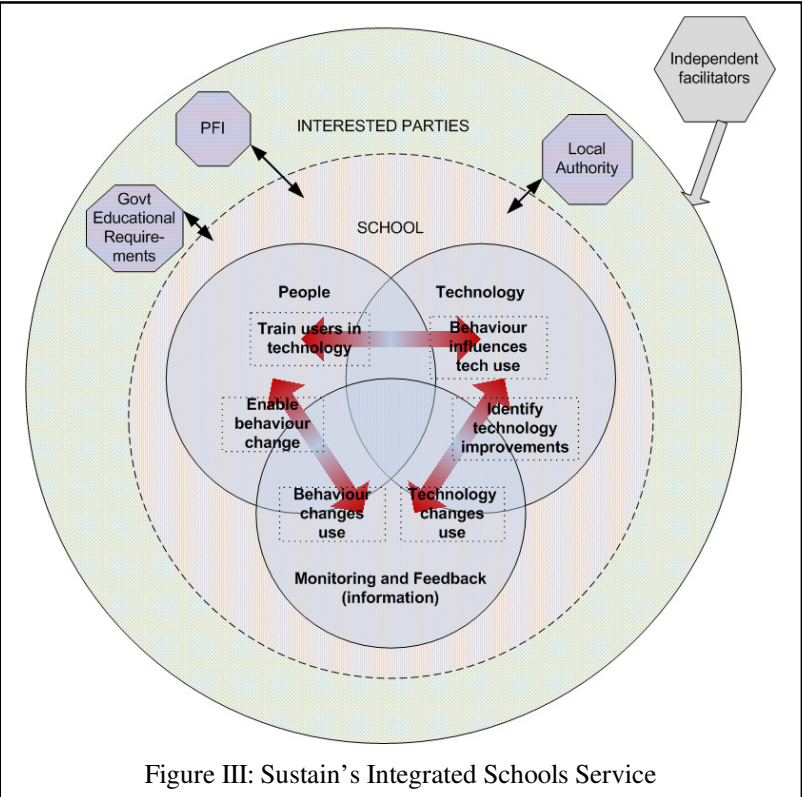

- $\quad$ Engaging all of the key stakeholder groups through an energy steering committee will ensure top-down support for any intervention and help to identify any policies that are leading to higher than needed energy use. It will ensure that end-user efforts are not impeded by top-down policies and top-down policies are not rejected by end-users.

- The benefits of this approach over a purely technical one are that any technology changes that are made will not be sabotaged by unexpected actions by end users, as can sometimes happen, and there will be more support and understanding towards facilities managers who are overseeing the building.

- The benefits of this approach over a purely behavioral one are that technology can be set up to be easy to switch off or monitor, ensuring people have control over equipment when needed. For example, in one school it was found that high electricity consumption was partly due to the fact that although the school was fitted with energy efficient lighting, the light switches required keys to be operated so lights remained on throughout the school day [14].

This approach will use soft systems methods to achieve the people side of the work, including stakeholder engagement, behavior change for end users, and the bringing together of facilities managers, staff, and management. It will draw upon existing established soft systems methodologies such as Checkland's SSM, but not follow them exactly due to the difference of focus compared to how these techniques are normally applied. The work will also use established systems engineering techniques to explore optimizations of end-use equipment such as IT and lighting, determine whether to use 
automated or manual controls, and evaluate the use of specialist equipment such as swimming pools.

\section{Conclusions}

This paper has explored the applicability of ST frameworks and methodologies to the wicked problem of reducing energy demand. The need to reduce energy demand has been firmly established but progress so far has been limited, and it is postulated that a ST approach would address some of the weaknesses of current policies. The paper found that ST frameworks could be particularly beneficial in addressing two fundamental research questions: What the nature of energy demand is, and whether a systems approach produces better energy demand reduction interventions than single interventions.

After defining the System of Interest (SoI) the MLP was explored as a way to address the first research question. It could be used to address uncertainty about the source of energy demand by conceptualizing the system as a sociotechnical regime within a larger sociotechnical landscape. Working to reduce demand can be aided by fully understanding how transformation happens within this regime. At the level of households and organizations, it is necessary to identify physical subsystems such as heating and lighting systems, identify soft subsystems such as the organization and the people who use the building, and also to also see the building and its users as a whole system (soft and hard combined). Some hard (SE) and soft (Checkland, Snowden, action research) methods were offered as candidate methodologies to do this, and examples were given of subsystems with different problem contexts. The paper concludes with a case study into the use of ST at Sustain Ltd, a carbon reduction company. Their low carbon buildings group are developing an integrated approach that includes energy information feedback, behavior change, and technology optimization, using both hard and soft systems methodologies. This service will meet the need to go beyond purely technical or purely behavioral solutions.

\section{Acknowledgements}

This work was supported by the Systems Centre at the University of Bristol, UK; the EPSRC funded Industrial Doctorate Centre in Systems (Grant EP/G037353/1); and Sustain Limited, Bristol. Dr Chris Preist, Toby Parker MBA, and Dr Theo Tryfonas were advisors.

\section{References}

[1] F. Geels and J. Schot, "Typology of sociotechnical transition pathways", Research Policy, vol. 36, pp. 399-417, 2007.
[2] DECC, "The UK Low Carbon Transition Plan: National Strategy for Climate and Energy", D. f. E. a. C. Change, 2009.

[3] J. Conklin, "Wicked Problems and Social Complexity", in Dialogue Mapping: Building Shared Understanding of Wicked Problems, Wiley, 2005.

[4] DECC, "Energy Consumption in the UK Overall Data Tables, 2010 Update", D.o.E.a.C. Change, Ed., 2010.

[5] C. Wilson and H. Dowlatabadi, "Models of Decision Making and Residential Energy Use", Annual Review of Environment and Resources, vol. 32, pp. 169-203, 2007.

[6] H. Wilhite, E. Shove, L. Lutzenhiser and W. Kempton, "Twenty Years of Energy Demand Management: We Know More About Individual Behavior But How Much Do We Really Know About Demand?", American Council for an Energy Efficient Economy (ACEEE) Summer Study on Energy Efficiency in Buildings, Pacific Grove, CA, USA, 2000.

[7] M. C. Jackson and P. Keys, "Towards a System of Systems Methodologies," The Journal of the Operational Research Society, vol. 35, 1984.

[8] M. C. Jackson, "Beyond a System of Systems Methodologies", The Journal of the Operational Research Society, vol. 41, pp. 657-668, 1990.

[9] A. Prodromou, A. Dasgupta, B. Jaffar, L. Curtis, M. Mulik, L. Chatzinasiou, G. Caruana, C. Demanuele, and D. Mumovic, "Consultation on the School Carbon Management Plan: UCL Evidence", London 2009.

[10] P. Checkland and J. Scholes, Soft systems methodology in action. Chichester: Wiley, 1990.

[11] J. Chapman, "System failure: Why governments must learn to think differently", Demos, www.demos.co.uk, 2004.

[12] C. F. Kurtz and D. J. Snowden, "The new dynamics of strategy: Sense-making in a complex and complicated world", IBM Systems Journal, vol. 42, 2003.

[13] P. Reason, G. Coleman and D. Ballard, "Insider Voices: Human Dimensions of Low Carbon Technology", Project report, University of Bath, UK, 2009.

[14] D. R. Gupta and S. Chandiwala, "Post Occupancy Evaluation, City of London Academy"; part-funded by the GROW EU Interreg3C joint programme, London 2007. 